\title{
Do Students See the "Selection" in Organic Evolution? A Critical Review of the Causal Structure of Student Explanations
}

\author{
Abhijeet Bardapurkar
}

Published online: 3 June 2008

(C) Springer Science + Business Media, LLC 2008

\begin{abstract}
This paper critically reviews and characterizes the student's causal-explanatory understanding; this is done as a step toward explicating the problematic of evolution education as it concerns the cognitive difficulties in understanding Darwin's theory of natural selection. The review concludes that the student's understanding is fundamentally different from Darwin's, for the student understands evolutionary change as necessary individual transformation caused by the transformative action of various physical and behavioral factors. This is in complete contrast to Darwin's (and even the Darwinian's, for that matter) understanding of evolutionary change as a change caused by accumulative selection. Hence, to understand natural selection, the student has to learn to "see" how the accumulative selection causes evolutionary change.
\end{abstract}

Keywords Understanding · Natural selection · Causality ·

Explanation $\cdot$ Evolution education $\cdot$ Darwin

This paper critically reviews the empirical work of science education researchers on the student's conceptions about organic evolutionary phenomena. The paper presents a spectrum of causal structures of the student's explanations from various studies. The effort is to interpret and organize the student's conceptions to see how she understands causality in evolutionary phenomena. This is a step toward explicating the problematic of evolution education as it concerns the cognitive difficulties in understanding Darwin's theory of natural selection. I specifically review the evolution

\footnotetext{
A. Bardapurkar $(\bowtie)$

Homi Bhabha Centre for Science Edu,

Tata Institute of Fundamental Research,

Post Box No. 8315, Deonar PO,

Mumbai 400 088, India

e-mail: abhijeet@hbcse.tifr.res.in
}

education literature that engages with the student's explanations ${ }^{1}$ of adaptive evolutionary phenomena (like the ones instantiated in artic fox's fur, cheetah's legs, bacteria's resistance, blind cave salamanders, deciduous trees, etc), and characterize the diversity of causal structures of these explanations.

\section{A Brief of How Natural Selection Explains Evolutionary Change}

Here, my goal is not to attempt a complete characterization of the causal-explanatory structure of Darwin's theory of natural selection. Instead, I lay out some of the relevant causal-explanatory features of the theory. These will constrain the following critical review by providing the necessary interpretive framework with which the student's causal explanations (documented in the literature) are to be contrasted and characterized.

Darwin's theory is a causal-explanatory narrative of how a least distinct but slightly adaptive stage of the (individual) difference changes into a more distinct and greatly adaptive stage of the (evolutionary) difference. It tells us how evolutionary change or "passage from one stage of difference to another and higher stage" (Darwin 1859/ 1964) is possible. A population of individuals could be changed in two fundamentally distinct ways ${ }^{2}$ : either by a

\footnotetext{
${ }^{1}$ This will have a limitation of leaving out some important works in the broader area of evolution education, like the works that employ concept mapping techniques and the ones like that of Anderson et. al. on the "Development and evaluation of the Conceptual Inventory of Natural Selection" published in 2002 in the Journal of Research in Science Teaching, Volume 39, pages 952-978.

2 There is a third, and perhaps even more fundamental, way- "Creation from scratch," but I will set that one aside for the present purpose.
} 
cause that acts on the individuals, thus transforming them; or by a cause that selects some of the slightly transformed individuals, thus accumulating them. I call the former change by transformative action, and the latter change by accumulative selection $^{3}$. Selection does not change the existing individual entities, there is no transformative action on the individuals; one is just preserving and accumulating what is available. In contrast, in transformative action, one is changing what is available. Selection preserves the existing individual change, whereas transformation causes the change.

To understand selection as a cause of evolutionary change, one must recognize the slight individual variation, "see" the variation being selected, and then see it being naturally accumulated in successive generations. The variation is naturally selected (i.e., preserved) because of its usefulness to the individual in its survival or reproduction-it is a natural consequence of the variation's advantageousness for the variant. Natural selection of better and better variation in successive generations results in the "accumulation" of the variation. The evolutionary change effected in selection is thus an accumulative change. To understand the how of selection, we understand the how of accumulation: we ask how the existing hereditary individual change (i.e., variation) is accumulated, or better, how the existing individual variation is naturally accumulated. A natural accumulation of a variation is a consequence of: one, the causal contribution of the variation in the variant's survival; and two, the variation's inheritance. In other words, a variation is naturally selected and accumulated because it proves to be profitable in the survival of the variant and it is hereditary. This accumulative selection explains the grand consequence of the evolutionary process that goes by the name of evolutionary adaptation.

Given this characterization of how the causal process of natural selection explains evolutionary change, now we begin to critically review how the student understands the causality of organic evolutionary events. In this paper, the "student/s" refers to all the subjects whose explanatory understanding has been documented in various contexts in the science education research literature.

\section{The Necessitating Necessity: "Need-Driven" Adaptive Change}

Very often, the student thinks that an organism "needs" to do an adaptive act (say, by repeatedly using a particular body structure) or "needs" to develop an adaptive trait to

\footnotetext{
${ }^{3}$ This distinction has its roots in Lewontin's (e.g. Lewontin 1984) distinction between "transformational" and "variational" evolution.
}

survive and flourish in its conditions of life. To her, the "adaptive" response or the "adaptive development" is a necessity in the current conditions. This necessity, along with the conditions that have contributed to it, necessitates the necessary adaptive evolutionary change, and thus forms a major explanatory concept in the student's worldview. The explanatory conception of the "necessitating necessity" manifests itself in various causal forms in the student's explanation of various situations. Let's look for these causalexplanatory manifestations documented in the literature.

The study by Demastes et al. $1996^{4}$ clearly brings out a role that the conception of "need" plays in the student's understanding of evolutionary change, particularly the role "need" plays in causing evolutionary change for better adaptation of the organisms to their environmental conditions. In fact, the authors of this study term the needbased conception of evolutionary change as a "controlling conception." They say that need "plays an important role in the learner's conceptual ecology for evolution" (p. 416). For example, a student (labeled as 'student M') in this study gives following response to "questions about evolution of webbed the feet in a population of ducks": "The trait of webbed feet in ducks... appear in ducks because they lived in water and needed to swim... webbed feet allows better swimming. It was an evolved trait, wasn't it? And it's not a chance mutation, it was something necessary. [Things evolve] mainly as far as when they need to, ah adapt to certain conditions" (p. 419; my emphasis). In this student's view, the evolution of webbed feet was necessary for better swimming and was caused "because [the ducks] lived in water" (ibid.; my emphasis). Thus, here you find that, to this student, the "need" of better swimming would ultimately explain the evolution of webbed feet-organisms need to change to be better adapted to the existing conditions in which they are living; but this need has arisen because of the conditions in which the organism finds itself, and these conditions are said to be an immediate cause of the adaptive change. This student (Student M) gives a similar need-based explanation in the case of evolution of running species in a population of Cheetahs: "[Cheetahs] needed to run faster... It was necessary for them to catch their pray to survive... it occurred because of the need for the adaptation... they needed to run faster to catch food so it happened" (p. 415). In this case, the necessitated adaptive act (faster running) has a particular goal-food! And the animal "had to" develop an adaptive trait if it is to survive. This develop-

\footnotetext{
${ }^{4}$ This study tracks the patterns of changes in the conceptual frameworks of a few students over a period of one year. Its goal was to understand "how students come to understand evolution" (p. 410) during a year-long biology course covering a variety of biology topics, but with a focus and a 10-day formal unit on evolution.
} 
mental tone is apparent also in a student's explanation in Jensen and Finley's 1995 study $^{5}$ : "As means for survival they had to catch their prey (so) they developed and learned how to increase speed over a number of years" (p. 156). Jensen and Finley call it an instance of "evolution on demand" conception. Such conceptions are also evident when students are thinking in the context of plants. For example, Ferrari and Chi 1998 quote the following response: "If the tree is to survive in the environment of the field, it will have to develop traits that are conductive to the amount of sunlight, water, parasites, etc., so it can continue to flourish" (p. 1245; emphasis in original). Such need-based and goal-directed explanations are also classified as teleological or functional ${ }^{6}$ explanations in the evolution education literature. Before we discuss instances of these in the literature, let us see another causal form that a need-based explanation takes in the student's thinking.

We saw a few instances of the student's need-driven explanations where a need, created by the environmental conditions, necessitates adaptive developmental change in the whole population. A need can also necessitate use or disuse of a body structure causing its modification and thus the evolutionary change. Examples of this are found in the student's explanation of changes in cave salamanders, in the studies of Bishop and Anderson 19907 , Settlage 1994 ${ }^{8}$, and Demastes et al. 1996. Just to illustrate, let us again take an example from the study of Demastes et al., when a student (student ST) is responding to a pretest question "about the evolution of blindness in a population of cave salamanders". She says: "the salamanders live in complete darkness, light is not a necessity... the ones that originally went into the cave became blind and their offspring were born without sight and they became blind and may be the process just happened quicker because they could function without sight... they don't use it, they lose it... ... [After five minutes she adds the following]... they became blind

\footnotetext{
${ }^{5}$ This study evaluates effectiveness of a "historically rich teaching innovation" (p. 147), in changing the students' non-Darwinian conceptions of evolution to the Darwinian conception.

${ }^{6}$ There are, however, important differences between teleological and functional explanations. For example, in one interpretation, functional explanations are non-causal explanations (photosynthesis might explain the presence of chlorophyll in a plant, but it is not a cause of the production of the pigment). In another interpretation, functional explanations could be causal without being teleological (chlorophyll pigment exists today because it has been performing the useful function of photosynthesis in the all the past instances). For an accessible and elegant overview, see Psillos 2007.

${ }^{7}$ Bishop and Anderson studied 110 college students enrolled in successive terms in their "nonmajors' introductory biology course" (p. 416).

${ }^{8}$ Settlage reports his study of 50 students (grade 9 to grade 12) done before and after a course titled "Evolution and Life on Earth." Each test had two similar essay questions.
}

just because [they] didn't need their sight. But I don't see how that could be passed on". But the students do not always offer this type of explanation. While talking about the evolution of running speed in a population of cheetahs, this student seems to be doubtful about the need-driven change: "I really don't think we can form something just because we need it" (p.416-7; my emphasis). In any case, even if the student is doubtful about the correctness of her understanding, we, at the least, can safely presume that the need-driven explanations are a kind of default explanation given by the students ${ }^{9}$ - that is, when no other convincing explanation is available to them, they are most likely to understand the change as necessitated by the "need" or the "conditions" of the time.

The examples in the preceding paragraph illustrate that the survival necessity and the conditions contributing to it are not distinct causal factors from the use/disuse of certain body parts; and hence I do not put "use/disuse" as a separate casual category in the student's explanation. Use/ disuse could, however, be quite a dominant causal explanatory factor in the student's view. In Bizzo's 1994 study ${ }^{10}$, students had the option of choosing either selection or use-disuse as an answer and defending it. In one case, an option of choosing artificial selection of the differences in the bone weights of the ducks, and in the other an option of choosing natural selection of a few faster felines present in some generations, was available to them. The author mentions the difficulty students face even after the apparent recognition that one of the options is Darwinian. For example, a student is quoted as writing: "both [the options] are correct because one is Darwinism, but cheetahs had to improve in every generation a little bit" (p. 541).

The student, when asked about the how of the evolutionary phenomena, refers not just to the "needs" and "wants" and the physical changes in the environment of animals as a cause of the change, but also to some "internal force" (Deadman and Kelly 1978). Thus, in the student's explanations, the causal factors are not restricted to the external "forces". In Banet and Ayuso's 2003 study, Spanish students (ages 14-16) believed that mutations take place to help organisms survive in unfavorable conditions; organisms respond to the environmental changes by mutating and thus attempting to avoid the possibility of extinction. A similar finding was reported by Brumby in her papers (e.g., Brumby 1979). She studied 63 (age about 18 years) British students, 47 of whom had studied evolution and heredity. The students categorized as having "poor" understanding of

\footnotetext{
${ }^{9}$ The general idea that in the case of children a kind of explanation could be a default explanation is from Gutheil et. al (Gutheil et al. 1998).

${ }^{10}$ Students in this study have already had formal instruction in the theory of evolution. They were aged between 15 and 17 years.
} 
natural selection $(65 \%)$ thought that the environment induces individual change (or "mutations") that makes organisms "immune" (possibly to the antibiotics or insecticides), thus adapting them to the environmental changes. For these students, adaptation is a process entirely governed by the environment. We should, hence, note that need does not always enter into the student's narrations as a causal factor, nor is it always associated with environmental conditions as a cause of adaptive transformation. Environmental factors alone are sometimes sufficient to bring up an adaptive change.

\section{Teleological or Functional Explanations}

In the preceding subsection, I clarified that I classify the commonly-referred-to category of "change by use/disuse" under what I have called "necessitating necessity" or "needdriven" adaptive development. In a similar way, a commonly used category of "teleological or functional explanations" is also entangled with what I have broadly delineated as the category of "need-driven explanations"11.

The "necessitating need" could be conceived by the student as an adaptive end toward which an individual's development is directed. Such a conception has a teleologicalexplanatory structure in which the end necessitates its beginning. For example, if having a thickly furry skin is conceived by the student as a "need" toward which the organism's development would be necessarily directed, she could easily be classified as having a "teleological" conception of organic change. An example of this, for instance, is found in Clough and Wood-Robinson (Clough and Wood-Robinson 1985b; they interviewed 84 English (UK) students (aged between 12 and 16 years). Most of the categorized responses from the younger students and about half of the responses from 16-year-olds were anthropomorphic and/or teleological. For many of these students, adaptive change is a conscious response of the organism driven by the need to survive in the changing or drastic environment. For example, when asked to explain how the thick coat of the Arctic Fox, which "lives well at very low temperature," came about originally, a 16-year-old girl said: "Fox at first when it had shorter fur would have been cold so it ... it knew that it had to change, really" (p. 127). A 12-year-old girl said: "gradually... [Foxes] began to grow thicker coats until they were able to survive properly ... yes, they were sort of determined to stay alive" (p. 127-8). Another 12-year-old said: "[An individual fox] grows thick coat, so it can keep warm while it's out looking for food" (p. 128).

\footnotetext{
${ }^{11}$ Here the reader is reminded of footnote 6. Although the categories of the student's explanation are overlapping with each other, there could be subtle and significant distinction among them.
}

The last response could fit the category of "functional explanations" as well - an individual has a trait for the function it serves. One of the difficulties in learning natural selection identified by Bishop and Anderson (Bishop and Anderson 1990) is the student's inability to distinguish between causal and functional explanations. For them, explaining the function of an organ is sufficient to explain its evolution ${ }^{12}$. However, the preceding subsection should remind us that 'purely functional' and 'naively teleological' is not always a hallmark of the student's explanation. For students' need-based explanations, although not purely mechanistic or natural, are still causal explanations in which the external physical conditions or internal forces act to bring out the necessary adaptive transformation. In an elegant piece of work, Tamir and Zohar 1991 pose a very interesting and important question: Do students use teleological and anthropomorphic terms just as convenient shorthand while they actually are aware of the differences between mechanistic-causal explanations, teleological explanations and anthropomorphic explanations? Or, do anthropocentric-teleological answers indeed reveal students' confusion between causal and teleological explanations? The authors randomly selected 12 grade 12 (age 17) and 16 grade 10 (age 15) students for this study.

When asked explicitly, many students in Tamir and Zohar's study could easily recognize anthropomorphic formulations (especially in the case of plants) as different from anthropomorphic explanations. In fact, grade 12 students even supported the inclusion of anthropomorphic statements in the textbook for the reasons of convenience and ease. But, at the same time it may be noted that only 3 of 28 students could give purely mechanistic explanations; and $62 \%$ of the students believed that animals do "really wish, try and strive" (p. 61). Even when a process or behavior is re-described to the student by removing the benefit it earlier had, a majority of the students still gave teleological or partially teleological (teleological but not in all the instances) responses. For example, when the students were asked to predict whether or not a deciduous plant growing in a greenhouse would shed its leaves, about $70 \%$ of the students gave teleological answers. For example: "Perhaps shedding the leaves is needed to complete the life cycle," or "perhaps without dormancy it cannot bloom" ( $p$. 63). Thus, Tamir and Zohar found teleological reasoning to be more common among students than anthropomorphic reasoning. Teleological responses do not necessarily in-

\footnotetext{
${ }^{12}$ Being satisfied with the functional explanations can surely and significantly make the learning of natural selection difficult. Nevertheless, here I would like to mention that if one takes a full view of causality in natural selection, functional explanations do figure in it, and in an important sense. After all, a particular trait is selected for the function it serves and thus its continual existence is explained by the utility it has for the organism.
} 
volve humanlike, conscious, goal-directed behavior. Instead, they are based on "the belief in the functionality of the behaviors of living organisms, which is illustrated by major principles such as the adaptability of organisms to their environment and the complementarity between structure and function" (p. 66).

\section{Evolutionary Change is Necessary Individual Transformation}

In the preceding section, we saw that, for the student, the need explains the adaptive change in an individual in two distinct but complementary ways: first, it could be taken simply as a necessity in the sense that the individual needs a particular trait or modification to adapt to and survive in the conditions; or second, it could be taken as a necessitating necessity, that is the need that somehow causes the individual to have or develop the necessary adaptive trait. In the first case, the conception of causality could be more complex: in this complex causal form, the fact that some adaptive trait is "needed" is not sufficient to explain its development; need is essential in explaining adaptive development, but by itself it does not cause (that is necessitate) the individual to change. The necessary change is caused (or necessitated) by living conditions, or the habits, or the use/disuse, or even through some internal force or mutations, ${ }^{13}$ e.g., the cave salamander lost their unnecessary eyes, but the loss is caused by disuse. In the second case, however, the necessity itself is deemed to be directly responsible for the necessary change, e.g., artic foxes developed thick fur for it was a necessity ${ }^{14}$ (see the "Teleological or Functional Explanations" subsection). In either of the cases, irrespective of the student's causal conception of necessity in the evolutionary phenomena, the organic change is understood by the student as a necessary individual transformation. The student finds little difference between individual change and evolutionary change, and therefore the cause of individual change (i.e., individual variation) is the same as the cause of evolutionary changeorigin of variation among individuals directly accounts for the origin of variation among species. This explains why the student sees "need as the [cause of] origin of variation" (Demastes et al. 1996, p. 413); and also, why the evolutionary change is seen by the student as a transfor-

\footnotetext{
${ }^{13}$ Again, it may be the case that the conditions that are said to cause the necessary adaptive transformation are the ones that have contributed in creating that necessity-if being in water is causing the necessary development of webbed feet, the aquatic habitat has also contributed to the creation of the need for webbed feet.

${ }^{14}$ The development of thick fur in artic foxes was necessitated by the necessity of having it.
}

mation or development in the traits: it is "gradual change in the traits themselves" (Bishop and Anderson 1990) (p. 423). In Jensen and Finley's 1995 study, the students often express the idea of an "individual 'evolving' with time" (p. 163). It is indeed paradigmatic to the student to understand "evolutionary" change in terms of transformation of individuals, rather than in terms of selection of individuals: adaptive transformation is "getting used to", it is "an individual process of adjustment" (Bizzo 1994, pp. 542-3 and 544; also see Wood-Robinson 1994). For the student, individual changes mean that the individuals evolve or adapt to the changing environment; and as we have already discussed, this deterministic adaptive change or evolution is understood to be caused by the "need" of that change and/or as a result of the "changes in the environment," or sometimes even because of the subconscious efforts of organisms to improve themselves. In their excellent paper, Deadman and Kelly 1978 note that the students in their study rarely recognized the significance of slight modifications and their adaptive value. Students do use the words "extinction" and "survival", but just as "shallow explanations," without linking them "in any deeper sense to selection mechanism"15 (p. 10). We come across many occasions where the evolution is explicitly or implicitly understood by the student in terms of individual transformative/developmental change. Below I quote a transcript of a typical response from Geraedts and Boersma (Geraedts and Boersma 2006): "[When the foxes move to a much colder environment] ... their coat will gradually become thicker, to adapt themselves to the cold. To keep them warm, otherwise they won't survive ... [And their children] will already begin with a thicker coat, and their coat will become thicker still" (p. 861; my emphasis). The last statement of this student leads our discussion of the causal structure of the student's explanations to a point where we deal with the conception of inheritance of acquired characters.

\section{Inheritance of Individual Adaptive Change}

Various students conceive the causality of organic change variously. However different their conceptions may be, if the organic change is to exist and amplify across generations, then it needs to be transmitted across generations, and this brings us to the students' notions of inheritance. In general, younger children tend to think that characters acquired by an individual in its lifetime will be passed on to the offspring. Kargbo et al. 1980 have reported young Canadian urban children's (ages 7 to 13 years) views about

\footnotetext{
${ }^{15}$ A general discussion of "shallows" of explanation may be found in Wilson and Keil 2000.
} 
inheritance of characters. Younger children in this study generally believed in the inheritance of a character abnormality acquired by animals during one's lifetime, and for them the chance of inheriting an acquired character is greater if the new character has been acquired by the animal at a younger age. Very few children, however, believed that plants inherit acquired characters. Significantly, many younger children thought the inheritance to be controlled by environmental factors (sun, water, food, parental care, attention), or body parts (blood, teats, brain), or even nature. Compared to the younger ones, older children have a much more nuanced notion of inheritance. In this study, children aged above 10 yrs. thought the contribution of parental traits to be important - "it would depend on whom the child takes after" (p.144). For predicting traits of offspring, older children wanted to know the traits of both the parents and their ancestors.

In their study of 84 English (UK) students (aged between 12 and 16 years), Clough and Wood-Robinson 1985a found a majority of the students thought that the characters acquired during one's lifetime are not inherited. Many of these thought the inheritance of acquired characters to be simply "unnatural." For example, when asked to predict and explain if the baby of normal mice whose tails are chopped off would have a tail or not, a "12-year-old said that the babies would have tails 'because it's not natural, nature didn't make it happen"' (p. 306). Another 14-yearold student said that the babies will have tails because mice "had tails until he chopped them off its ... well ... they were born with tails, so the other mice would be born with tails" (p. 306). About 20-25\% students in this study did not believe in the inheritance of acquired characters because there is no genetic change in acquiring the character. A 12year-old boy said in answering the tail task that "they'd still have tails because the chromosomes wouldn't have altered -it was just the tails that had been chopped off' (p. 306). However, interestingly many of these students who thought that the acquired characters are not inherited did say that if organisms in each of the successive generations keep acquiring a character, the acquired character is inherited: the tail-cutting in each generation would "work in the end, given time" (p. 306). A 12-year-old boy said that if tails are cut repeatedly over many generations, then mice "probably wouldn't bother growing their tails any more if they knew they were just going to lose them" (p. 306). The idea that given enough time, acquired characters are inherited was found to be quite persistent and prevalent among the students. This belief is also common among Botswana students (Wood-Robinson 1994, p. 40). In the case of inheritance of athletic ability, compared to $13 \%$ of the UK student sample, $31 \%$ of the Botswana student sample believed in the inheritance of acquired athletic ability. However, a majority of the Botswana student sample did not believe in the inheritance of the acquired characters. Thus, talking about the farmer's calloused hands, one student said: "Children do not inherit features that the parent acquired, but only inherit those that they are actually born with. The parent's hands have nothing to do with what is in the womb" (p. 39).

From the students' responses, it is evident that whenever students have the standard concept of inheritance- - that is, whenever they think the structural gain or loss during one's lifetime is not inherited, the explanation is commonly not grounded in the understanding that the traits are dependent not on the living conditions but on the "genetic" factors. The awareness of the characters being determined by genetic entities may not ensure us of the understanding of non-inheritance of acquired characters. Evidently, it is not very difficult for the students to entertain the possibility that just as body structures are, "genetic" structures could as much be adaptively transformed and inherited. For instance, in Lawson and Thompson's (Lawson and Thompson 1988) study, when asked about the skin color of the child of a fairskinned girl who grew up in Africa and then married a man of the same race, living in Africa thereafter, a student in this study said: "Probably somewhat darker because the mother's chromosomes have adapted" (p. 739; my emphasis). The changes could thus be acquired at the "genetic" level too, leaving little doubt that the acquired skin color is inherited. Even if students have an idea that the trait is not always conditioned by environmental factors and that they are in some way dependent on DNA, they accommodate this fact to the framework that predicts inheritance of acquired characters. A response by one of the students in Bizzo's 1994 study supports this ${ }^{16}$ : "[In circumcision] having removed the DNA of that part for many generations, it disappeared" (p. 541). It is clear that having an idea of "gene"-controlled inheritance is not enough, neither does it appear to be very useful, in understanding that evolution is not the inheritance of necessary adaptive transformations.

\section{Conclusion}

The student understands evolutionary change as an individual transformation. The individual transformation is commonly explained by perceiving its need/necessity - the transformative change happens because it is a necessity. The explanatory perception of necessity, however, could assume a variety of causal forms in the student's explanations. In some instances, a necessity or a need could be perceived as an immediate cause of the necessary change,

\footnotetext{
${ }^{16}$ Many students (about or more than half) in this study believed in the inheritance of acquired characters.
} 
but sometimes the need is deemed explanatory without any reference to the cause of the change. In other instances, the student does perceive the necessity of the change, but a distinct factor is identified as a cause of the change. The causal factors could be the physical conditions (the conditions that have contributed to the creation of the need in the first instance), and they can bring in the necessary change by transforming the structural or genetic traits of the individual. Or, the causal factor could even be some internal force. Moreover, there are many instances when the necessary change is caused by the corresponding use/ disuse of the body parts. The adaptive individual transformation achieved through the conglomeration of this variety of the necessitating/causal factors could be inherited by the coming generations, but the inheritance again depends on a range of conditions such as the age at which transformation occurs, the number of generations that have been subject to it, whether or not chromosomes or genes are also transformed in the process, etc.

It is clearly evident that the students rarely "see" the evolutionary change being effected by accumulative selection. For the student, evolutionary change is caused by transformative action. In fact, the student finds little difference between individual change and evolutionary change, and therefore the cause of individual change (i.e., individual variation) is the same as the cause of evolutionary change - the origin of variation among individuals directly accounts for the origin of variation among species. The teachers have to note that the student's understanding is fundamentally different from that of Darwin's: Darwin naturalized the selection, whereas the student naturalizes the transformation. To understand natural selection, the student has to learn to see: first, the difference between individual change and evolutionary change and then, the difference between a cause that effects evolutionary change by transformative action and a cause that effects evolutionary change by accumulative selection.

Acknowledgments Many thanks to G. Nagarjuna and Arvind Kumar for their comments on an earlier draft of this paper.

\section{References}

Banet E, Ayuso GE. Teaching of biological inheritance and evolution of living beings in secondary school. Int J Sci Educ. 2003;25(3):373-407.

Bishop BA, Anderson CW. Student conception of natural selection and its role in evolution. J Res Sci Teach. 1990;27(5):415-27.

Bizzo NMV. From down house landlord to Brazilian high school students: what has happened to evolutionary knowledge on the way? J Res Sci Teach. 1994;31(5):537-56.

Brumby M. Problems in learning the concept of natural selection. J Biol Educ. 1979;13(2):119-22.

Clough EE, Wood-Robinson C. Children's understanding of inheritance. J Biol Educ. 1985a;19(4):304-10.

Clough EE, Wood-Robinson C. How secondary students interpret instances of biological adaptation. J Biol Educ. 1985b;19(2):125-30.

Darwin C. On the origin of species: A facsimile of the first edition. Cambridge, MA: Harvard University Press; 1964. p. 52. (Original work published 1859).

Deadman JA, Kelly PJ. What do secondary school boys understand about evolution and heredity before they are taught the topics? J Biol Educ. 1978;12(1):7-15.

Demastes SS, Good RG, Peebles P. Patterns of conceptual change in evolution. J Res Sci Teach. 1996;33(4):407-31.

Ferrari M, Chi MTH. The nature of naïve explanations of natural selection. Int J Sci Educ. 1998;20(10):1232-56.

Geraedts CL, Boersma KT. Reinventing natural selection. Int J Sci Educ. 2006;28(8):843-70.

Gutheil G, Vera A, Keil FC. Do houseflies think? Patterns of induction and biological beliefs in development. Cognition. 1998;66:3-49.

Jensen MS, Finley FN. Teaching evolution using historical arguments in a conceptual change strategy. Sci Educ. 1995;79(2):147-66.

Kargbo DB, Hobbs ED, Erickson GL. Children's beliefs about inherited characteristics. J Biol Educ. 1980;14(2):137-46.

Lawson E, Thompson LD. Formal reasoning ability and misconceptions concerning genetics and natural selection. J Res Sci Teach. 1988;25(9):733-46.

Lewontin RC. The structure of evolutionary genetics. In: Sober E, editor. Conceptual issues in evolutionary biology. Cambridge, MA: Bradford/MIT Press; 1984. p. 3-13.

Psillos S. Philosophy of science A-Z. Edinburgh: Edinburgh University Press; 2007. p. 97-100.

Settlage J Jr. Conceptions of natural selection: a snapshot of the sensemaking process. J Res Sci Teach. 1994;31(5):449-57.

Tamir P, Zohar A. Anthropomorphism and teleology in reasoning about biological phenomena. Sci Educ. 1991;75(1):57-67.

Wilson RA, Keil FC. The shadows and shallows of explanation. In: Keil FC, Wilson RA, editors. Explanation and cognition. Cambridge, MA: MIT Press; 2000. p. 87-114.

Wood-Robinson C. Young people's ideas about inheritance and evolution. Stud Sci Educ. 1994;24:29-47. 